Article Type: Education and Training

Subheading: Gynecology

EDUCATION AND TRAINING

\title{
Standardizing bimanual vaginal examination using cognitive task analysis
}

Isabella Plumptre ${ }^{1,{ }^{*}}$, Omar Mulki ${ }^{2}$, Alejandro Granados ${ }^{1}$, Claudine Gayle ${ }^{1}$, Shahla Ahmed $^{2}$, Naomi Low-Beer ${ }^{2}$ Jenny Higham $^{3}$, Fernando Bello ${ }^{1}$

${ }^{1}$ Faculty of Medicine, Imperial College, London, UK

${ }^{2}$ Imperial College Healthcare NHS Trust, London, UK

${ }^{3}$ St George's, University of London, London, UK

${ }^{*}$ Corresponding author: Isabella Plumptre

Imperial College Centre for Engagement and Simulation Science, Academic

Surgery, Chelsea and Westminster Hospital, London, UK. Email address:

bella.plumptre@gmail.com

Keywords: Bimanual vaginal examination; Cognitive task analysis; Education;

Pelvic Simulation; Training

Synopsis: Through novel pelvic simulation and cognitive task analysis, bimanual vaginal examination was deconstructed into a core schema of 30 standardized steps.

This article has been accepted for publication and undergone full peer review but has not been through the copyediting, typesetting, pagination and proofreading process, which may lead to differences between this version and the Version of Record. Please cite this article as doi: 10.1002/ijgo. 12260

This article is protected by copyright. All rights reserved. 


\section{Abstract}

Objective: To create a standardized universal list of procedural steps for bimanual vaginal examination (BVE) for teaching, assessment, and simulator development. Methods: This observational study, conducted from June-July 2012 and JulyDecember 2014, collected video data of 10 expert clinicians performing BVE in a nonclinical environment. Video data were analyzed to produce a cognitive task analysis (CTA) of the examination steps performed. The CTA was further refined through structured interviews to make it suitable for teaching or assessment. It was validated through its use as a procedural examination checklist to rate expert clinician performance.

Results: BVE was deconstructed into 88 detailed steps outlining the complete examination process. These initial 88 steps were reduced to 35 by focusing on the unseen internal examination, then further refined through interviews with five experts into 30 essential procedural steps, five of which are additional steps if pathology is suspected. Using the CTA as a procedural checklist, the mean number of steps performed and/or verbalized was $21.6 \pm 3.12(72 \% \pm 10.4 \%$; range, 15.9-27.9, $53 \%-93 \%)$.

Conclusion: This approach identified 30 essential steps for performing BVE, producing a new technique and standardized tool for teaching, assessment, and simulator development.

\section{Introduction}

Bimanual vaginal examination (BVE) is an essential clinical skill and key for the diagnosis of various gynecological pathologies [1-3]. Despite its importance, medical students report a lack of experience, training, and confidence in performing the 
procedure [4-6]. Lack of clinical examination proficiency can be detrimental to the safety of the patient and their healthcare experience [7].

Teaching BVE is problematic. Firstly, the examination is "blind" in that internal finger movements cannot be visualized by the student or teacher. Students rely on the teacher's ability to verbalize their actions; however, some processes may be unconscious and experts are poor at verbalizing the steps [8]. Secondly, the BVE process is not standardized [7] and it may vary across subspecialties, which may result in students missing key steps for a safe examination, as well as adding more advanced steps that are beyond their educational needs and capabilities. Thirdly, obtaining clinical experience is increasingly difficult [9] as patients are reluctant to undergo intimate examinations for teaching purposes. Training with gynecological teaching associates is a viable alternative [10], but can be expensive. As a consequence, education is shifting toward simulation-based training.

Cognitive task analysis (CTA) is a method of capturing expert knowledge by breaking down a process into its fundamental steps, including elements performed unconsciously [11]. Recent applications of CTA in medical education include developing simulation and teaching materials $[12,13]$, which show improved learning outcomes for technical skills $[14,15]$.

This study used a novel bench-top pelvic simulator to decode expert knowledge of BVE. The primary aim was to outline the sequence of steps needed to perform BVE. Secondly, we aimed to organize the information into an accessible and useful tool for 
future application in teaching, assessment, and/or development of haptic (touch feedback) simulators.

\section{Materials and methods}

This observational study recorded expert clinicians performing BVE in a nonclinical setting at Imperial College London, UK, from June-July 2012. To generate a CTA, video and audio data were collected from 10 subject matter experts (SMEs) who performed BVE on a bespoke pelvic model. A sample size of 10 was considered reasonable in comparison with similar studies [12]. SMEs were defined as qualified obstetrician-gynecologist UK consultants (US attending) with at least 10 years of clinical experience in women's health [16] and regular teaching responsibilities for medical students and/or residents. They were recruited from the obstetrics and/or gynecology departments at Imperial College Healthcare NHS Trust and gave informed consent for examination recording. Ethical approval for the study was obtained from the NHS National Patient Safety Agency Research Ethics Committee (09/H0701/68).

To record the internal examination steps a new pelvic bench-top model (Studiohead, London, UK) was produced with an acrylic frame, silicone vulva, semitransparent silicone vagina, and polyurethane foam uterus and ovaries (Figure 1). In contrast to traditional commercially available models, in this model soft materials are used to better replicate physical tissue. The uterus is suspended by silicon-based ligaments for a more realistic anatomical feel. The ovaries attach to the anterior abdominal wall at various positions for different training scenarios. Two high-definition cameras mounted inside the model (anterior and lateral to the vaginal canal) allowed direct 
visualization of internal finger movements (Figure 2). A third, tripod-mounted camera captured an external view.

CTA was performed in three stages. The first stage, conducted from June to July 2012, generated a CTA for BVE. The second refined this detailed CTA to form a checklist suitable for teaching or assessment. The third stage evaluated the CTA to confirm that the essential examination steps were successfully captured. The last two stages were conducted from July to December 2014.

SMEs each performed two BVEs on our prototype model. According to methods previously described for digital rectal examination [12], the bench-top model was combined with a head and neck mannequin and covered with a blanket to simulate a normal clinical environment. For the first examination, SMEs were instructed to examine and interact with the model as would be done during a routine gynecological examination. For the second examination, the SMEs were asked to interact with a researcher present in the room as though they were a medical student who was unfamiliar with BVE and needed to be taught best practice. Pressure and positioning sensors on the examiner's fingertips [17] collected force and trajectory data for potential future use. The first examination lasted less than 2 minutes as per standard practice. The second examination with researcher interaction took slightly longer owing to the verbal explanations that were provided (2-3 minutes).

SMEs answered a post-examination questionnaire about model limitations, the role of different examination aspects including communication, and questions to inform on the potential uses of the CTA. 
The resulting full list of steps described every aspect of BVE in detail. However, from an educational perspective recognizing the potential of a CTA checklist for teaching and assessment, this initial CTA was deemed too long to be of practical use. Furthermore, many aspects (e.g. communication steps) are already covered extensively elsewhere in medical education. The more challenging parts of BVE are the unseen internal steps, which are also critical to develop computerized simulation models. Accordingly, we decided to focus our CTA to comprehensively describe the internal examination steps, at a level appropriate for medical student teaching. With this aim, video and audio data were reanalyzed. A reduced-step CTA was produced with more detail provided on the internal examination. These steps were written so that a medical student with basic anatomical knowledge but no previous experience of BVE could replicate the examination with no additional input.

This reduced-step CTA was assessed and further refined by five additional independent SMEs (as previously defined) through structured interviews. A sample size of five was deemed appropriate based on the related literature [12,14]. SMEs were asked to justify appropriate and inappropriate steps, clarify dissenting performances, and identify critical steps to ensure safe practice at medical student level. For example, following expert feedback, the step detailing insertion of digits into the vaginal canal was edited from "two" to "one or two" digits. Although only one SME performed the examination with a single digit, SMEs stated that juniors should always consider inserting one finger initially, before later inserting two according to necessity and patient comfort. Any disagreement was reconciled through further independent discussions until full agreement was reached on a final refined checklist, paralleling similar studies [12-14]. 
The last stage validated the final CTA to assess if it captured the essential steps of BVE successfully. To do this, the original SME examination videos were reanalyzed and scored against the refined CTA. This determined the percentage of steps that were clearly observed and/or verbalized, and those that were not clearly performed (either missed by the clinician, or unclear from limitations in the simulation and camera set-up). Scoring was undertaken by one author (OM, Rater 1) and a separate independent expert (Rater 2). Rater selection was done in line with similar studies [12,14]. SPSS version 22.0 (IBM, Armonk, USA) was used for statistical analysis.

\section{Results}

Video and audio data of examinations performed by the 10 SMEs was analyzed to produce a comprehensive list of 88 steps (Supporting information) [16]. This took a holistic view of BVE, including communication, preparation, and subspecialty specific steps. Although globally comprehensive, the steps were not specific enough for a student to replicate the examination, were too long to be of practical use for teaching, and provided insufficient detail of unseen internal steps for input into digital simulation.

The initial 88-step CTA was reduced to 35 steps after reanalyzing video and audio data, focusing on internal aspects of BVE. Further refinement through structured interviews reduced the CTA to 30 procedural steps (25 essential, 5 deemed necessary only if pathology was noted) (Table 1) [18]. This 30-step CTA is more practical and in a more useful format for teaching and assessment, as well as for 
developing computer simulations. It consists of anatomically comprehensive steps that students can learn and replicate to perform BVE.

The final 30-step CTA was used as a procedural examination checklist to rate the videos of the expert clinicians. The sensitivity of the judgments between the two raters was $69 \%$. We observed fair agreement between the raters' judgments using Cohen's kappa, $\mathrm{K}=0.327(95 \% \mathrm{Cl}, 0.249-0.405), P<0.001$, and a moderate positive Spearman's rank-order correlation between the raters' judgments $r_{s}(298)=0.549$, $P<0.001$. The resulting concordance between the raters can be partly explained by differences in judgments when it was unclear for Rater 2 if optional steps were performed and Rater 1 was either able to see them or not (29 [9.6\%] cases). Yes/No disagreements accounted for only 22 (7.3\%) cases altogether (Table 2). Power analysis indicates that our sample size is adequate when we take into consideration this correlation coefficient as the effect size measure with a significance level of 0.05 .

The mean number of the 30 CTA steps performed and/or verbalized by SMEs was $21.6 \pm 3.12(72 \% \pm 10.4 \%$; range 15.9-27.9, 53\%-93\%) (Figure 3; left image). The steps that were most commonly missed were step 2 ("If concerned, palpate the areas of concern") and step 27 ("If endometriosis is suspected, insert the finger(s) deeper until the posterior fornix is felt and attempt to feel the uterosacral ligaments"). These were both performed by only one SME (10\%) (Figure 3; right image), and are both additional steps if pathology is suspected.

This article is protected by copyright. All rights reserved. 
From the post-examination questionnaire, 7 (70\%) SMEs strongly disagreed or disagreed that the bench-top model used had serious limitations, and 3 (30\%) neither agreed nor disagreed. Comments included that the ovaries were easier to feel than in reality, but that this might be beneficial for training purposes. The vulva, vagina, and especially the introitus were not quite accurate, while the mid- and upper vagina were deemed very accurate. The silicon materials were thought to make the model more realistic than current commercial models. The semitransparent vagina was deemed very useful, and it was suggested that this semitransparency could be incorporated into the entire model. It was felt that real-time visual feedback about hand position and anatomy would be very beneficial for students when learning, as well as for trainers to give feedback. Nine (90\%) SMEs strongly disagreed or disagreed that current BVE training is adequate. Ten (100\%) SMEs strongly agreed or agreed that virtual reality simulators would be useful training aids, and commented that the model would benefit teachers since they could assess if students had really felt the anatomical structures. Ten (100\%) SMEs strongly agreed or agreed that junior doctors lack confidence in performing BVE, and commented that simulation with haptic feedback would allow repeated practice, resulting in improved clinical performance.

\section{Discussion}

This study applied CTA methodology to successfully outline the necessary steps to perform a comprehensive and safe BVE. We generated and validated a 30-step procedural examination checklist for use in teaching (as a stand-alone tool, or in tandem with existing teaching), as an assessment mark scheme, or simulator development. Overall, SME examinations covered the majority (21.6 [72\%]) of steps 
in the finalized CTA. There were only minor discrepancies in missed steps, with steps most commonly missed only necessary if specific pathology is suspected. This demonstrates that our CTA successfully captures the steps in routine expert examinations, and justifies our refinement and evaluation. Subspecialist interest steps (e.g. gyne-oncologist lymph node palpation) were eliminated during CTA refinement, as such steps were deemed beyond the appropriate standard for medical students.

After successfully harnessing CTA methodology for digital rectal examination [12], we wanted to apply this approach to BVE_-another "unseen" internal examination. These are both core competencies expected of medical graduates [19], and skills that medical students report feeling underprepared to perform $[4,5,20]$. Furthermore, there is a lack of standardization and current guidelines do not specify or justify pelvic examination steps $[21,22]$. Thus, there is room for innovative teaching approaches to BVE to improve learning outcomes.

To the authors' knowledge, this is the first descriptive study to produce a standardized universal checklist of procedural steps in BVE. CTA methodology has proved beneficial in other examinations [12] and procedures [13-15]. Furthermore, CTA methodology in this study captures SME skill and knowledge through performance analysis and structured interviews, respectively. The resulting extended CTA of BVE depicts all aspects of the examination, including communication, while the refined CTA summarizes technical steps into a checklist suitable for teaching, assessment, and simulator development. The bench-top model designed for this study enables direct visual feedback of internal steps, which has never previously 
been possible. We plan to assess the CTA's effectiveness as a medical student teaching tool in a future educational study. Similar to previous work using haptic feedback for digital rectal examination [23] and rectal palpation of the bovine uterus $[24,25]$, there is also scope to apply our CTA data to develop a virtual reality haptic simulator for BVE.

The study has several limitations. Firstly, individual SMEs did not perform all steps in the resulting CTA. This could be interpreted as the CTA including unnecessary steps; however, five steps were only required if pathology was suspected, and these were the most commonly missed steps. Furthermore, the CTA's purpose was to reduce variability between clinicians by providing a standard schema for a thorough, safe examination. Secondly, SMEs only performed BVE twice, and were prompted to narrate their steps for the second examination. As they were prompted by a researcher and not a student, the descriptions and explanations may not reflect a traditional teaching session. In contrast to the digital rectal examination CTA [12], SMEs in the present study performed well at verbalizing internal steps. However, especially with awareness of recording, this level of description may not be representative of standard teaching sessions. Validity and concordance among raters could be improved with additional raters of SME performance. Finally, this study targeted BVE teaching at medical student level; a more complex CTA with a greater focus on identifying pathology and cognitive decision points would be necessary for specialty training.

This article is protected by copyright. All rights reserved. 
Three SMEs (30\%) neither agreed nor disagreed that there were "serious limitations" to the model. However, as the major criticism was that more robust materials should be used, this is unlikely to have had a serious impact on the overall examination steps performed, or on the resulting CTA. Regardless, simulator characteristics could be improved. Three-dimensional printing with translucent plastic would eliminate the need for electronics and cameras, and render the model more marketable and distributable. Studying recorded force and positional data could improve understanding of expert performance to provide benchmarks. Combined with a pressure sensor, this could prompt reactions from simulated patients if the examiner uses enough force to elicit discomfort.

Prior CTA research has targeted advanced procedures where finger movements can be visualized and may cause significant risk of physical harm to the patient $[8,13-$ 15]. Risks associated with BVE are related to patient distress and diagnostic failures rather than to physical harm. This is reflected in the CTA in that cognitive steps aim to recognize patient distress and respond appropriately, as well as elicit abnormal findings. Furthermore, BVE requires internal finger movements that neither student nor teacher can visualize, but were made explicit through our methodology. This ensures students are taught best practice and key pathological signs are not missed clinically, thus improving safety and patient experience [7].

In conclusion, this study used CTA to deconstruct BVE into a validated 30-step standardized procedural checklist for use in teaching, assessment, and simulator development. 


\section{Contribution to authorship}

Study concept and design: OM, AG, CG, SA, NLB, JH, FB. Acquisition of data: OM AG, CG, FB. Analysis and interpretation of data: IP, OM, AG, CG, FB. Drafting of manuscript: IP, OM, AG, CG, SA, NLB, JH, FB. Approval of final draft of manuscript: IP, OM, AG, CG, SA, NLB, JH, FB.

\section{Acknowledgments}

The authors would like to thank Niels Hald for his assistance with the study set-up, and to the expert clinicians who participated and assisted with the study.

\section{Conflicts of interest}

The authors have no conflicts of interest to disclose.

\section{References}

1. Stoelinga B, Huirne J, Heymans MW, Reekers JA, Ankum WM, Hehenkamp WJ. The estimated volume of the fibroid uterus: a comparison of ultrasound and bimanual examination versus volume at MRI or hysterectomy. Eur J Obstet Gynecol Reprod Biol 2014;184:89-96.

2. Shulman LP. New recommendations for the periodic well-woman visit: impact on counseling. Contraception 2006;73:319-24.

3. Singh RH, Erbelding EJ, Zenilman JM, Ghanem KG. The role of speculum and bimanual examinations when evaluating attendees at a sexually transmitted diseases clinic. Sex Transm Infect 2007;83:206-10. 
4. Pierides K, Duggan P, Chur-Hansen A, Gilson A. Medical student selfreported confidence in obstetrics and gynaecology: development of a core clinical competencies document. BMC Med Educ 2013;13:62.

5. Fairbank C. Effective teaching of pelvic examination. Clin Teach 2009;6:1014.

6. Abraham S, Chapman M, Taylor A, McBride A, Boyd C. Anxiety and feelings of medical students conducting their first gynecological examination. J Psychosom Obstet Gynecol 2003;24(1):39-44.

7. Bates CK, Carroll N, Potter J. The challenging pelvic examination. J Gen Intern Med 2011;26:651-7.

8. Sullivan ME, Yates KA, Inaba K, Lam L, Clark RE. The use of cognitive task analysis to reveal the instructional limitations of experts in the teaching of procedural skills. Acad Med 2014;89:811-6.

9. Hartz MB, Beal JR. Patients' attitudes and comfort levels regarding medical students' involvement in obstetrics-gynecology outpatient clinics. Acad Med 2000;75:1010-4.

10. Duffy J, Chequer S, Braddy A, et al. Educational effectiveness of gynaecological teaching associates: a multi-centre randomised controlled trial. BJOG 2016;123:1005-10.

11. Clark RE, Feldon D, van Merrienboer J, Yates K, Early S. Cognitive task analysis. In: Spector JM, Merrill MD, van Merrienboer J, Driscoll MP, eds. Handbook of Research on Educational Communications and Technology. Third ed. Mahwah, NJ: Lawrence Erlbaum Associates; 2008. 
12. Low-Beer N, Kinnison T, Baillie S, Bello F, Kneebone R, Higham J. Hidden practice revealed: using task analysis and novel simulator design to evaluate the teaching of digital rectal examination. Am J Surg 2011;201:46-53.

13. Sullivan ME, Ortega A, Wasserberg N, Kaufman H, Nyquist J, Clark R. Assessing the teaching of procedural skills: can cognitive task analysis add to our traditional teaching methods? Am J Surg 2008;195:20-3.

14. Velmahos GC, Toutouzas KG, Sillin LF, et al. Cognitive task analysis for teaching technical skills in an inanimate surgical skills laboratory. Am J Surg 2004;187:114-9.

15. Campbell J, Tirapelle L, Yates K, et al. The effectiveness of a cognitive task analysis informed curriculum to increase self-efficacy and improve performance for an open cricothyrotomy. J Surg Educ 2011;68:403-7.

16. Gayle C. Can a Cognitive Task Analysis be Developed That is Likely to be Useful for Teaching Vaginal Examination? [Master's thesis] London: Imperial College; 2013.

17. Granados A, Hald N, Di Marco A, et al. Real-time visualisation and analysis of internal examinations - seeing the unseen. Med Image Comput Comput Interv $2014 ; 17: 617-25$.

18. Mulki O. Cognitive Task Analysis - An Effective Teaching Tool for Bimanual Vaginal Examinations [Master's thesis] London: Imperial College; 2015.

19. Association of American Medical Colleges. Core Entrustable Professional Activities for Entering Residency. Curriculum Developers' Guide. Washington DC: AAMC; 2014. 
20. Fitzgerald D, Connolly SS, Kerin MJ. Digital rectal examination: national survey of undergraduate medical training in Ireland. Postgrad Med J 2007;83:599_ 601.

21. Saslow D, Runowicz CD, Solomon D, et al. American Cancer Society guideline for the early detection of cervical neoplasia and cancer. CA Cancer J Clin 2002;52:342-62.

22. ACOG Committee on Practice Bulletins -- Gynecology. ACOG Practice Bulletin no. 109: Cervical cytology screening. Obstet Gynecol 2009;114:1409-20. 23. Granados A, Mayer E, Norton C, et al. Haptics Modelling for Digital Rectal Examinations. In: Bello F, Cotin S, eds. Biomedical Simulation. Proceedings of the 6th International Symposium on Biomedical Education (ISBMS), Strasbourg, France. October 16-17, 2014. 8789:40-9. Switzerland: Springer; 2014.

24. Baillie S, Crossan A, Brewster S, Mellor D, Reid S. Validation of a bovine rectal palpation simulator for training veterinary students. Stud Health Technol Inform 2005;111:33-6.

25. Baillie S, Mellor DJ, Brewster SA, Reid SWJ. Integrating a bovine rectal palpation simulator into an undergraduate veterinary curriculum. J Vet Med Educ 2005;32(1):79-85.

This article is protected by copyright. All rights reserved. 


\section{[Note to typesetter: Please retain the red italics in Table 1]}

Table 1. Thirty-step cognitive task analysis of bimanual vaginal examination.

\section{Note: Any vaginal examination should be preceded by abdominal palpation. You must have a chaperone and patient consent.}

The text in black denotes the key and essential steps. The text in red italics denotes nonessential steps usually contingent on finding pathology/disease.

\begin{tabular}{|c|c|}
\hline 1 & $\begin{array}{l}\text { Make sure gloves are worn. Inspect the external genitalia making sure you view the vulva, } \\
\text { perineum, vestibular area, perianal area, and interlabial folds. }\end{array}$ \\
\hline 2 & If concerned, palpate the areas of concern. \\
\hline 3 & Using two fingers from the nondominant hand, separate the labia minora. \\
\hline 4 & $\begin{array}{l}\text { Making sure your finger is lubricated on your dominant hand, insert your index finger (+/- } \\
\text { middle finger) to the distal interphalangeal joint (finger in line with the sagittal plane). }\end{array}$ \\
\hline 5 & $\begin{array}{l}\text { If tactile sensation from the fingers reveals any abnormalities or if there is clinical concern, } \\
\text { palpate the inner vulva/introitus using pronation and supination movements until a } 360 \\
\text { degree sweep has been completed. }\end{array}$ \\
\hline 6 & $\begin{array}{l}\text { If appropriate (i.e. NOT in virgin females or tight introituses) and if not already done in step } \\
4 \text {, insert the middle finger in the anteroposterior diameter in line with the sagittal plane with } \\
\text { both fingers facing the wall of the vagina (index superior to the middle finger). }\end{array}$ \\
\hline 7 & $\begin{array}{l}\text { Continue insertion of the finger(s) aiming posterior and inferior; at the same time be } \\
\text { mindful of the tactile feedback from the finger(s) on all surfaces to detect any } \\
\text { abnormalities such as lumps, bumps, and changes in texture. As the insertion occurs, } \\
\text { rotate the fingers into the coronal plane so that the finger(s) are facing upward. }\end{array}$ \\
\hline 8 & $\begin{array}{l}\text { If any abnormality is suspected or detected, with the fingers fully inserted rotate the fingers } \\
\text { using pronation and supination movements to palpate every wall of the cavity using a } 360 \\
\text { degree rotation. }\end{array}$ \\
\hline 9 & $\begin{array}{l}\text { Adjust finger(s) position until the posterior-inferior edge of the cervix is felt (finger(s) in } \\
\text { coronal plane facing upward). }\end{array}$ \\
\hline 10 & $\begin{array}{l}\text { At the cervix, use the finger(s) to feel the inferior surface and cervical os (assessing for } \\
\text { lumps or bumps). }\end{array}$ \\
\hline 11 & $\begin{array}{l}\text { Place the finger(s) on the posterior edge of the cervix (finger(s) to overlap with cervix by a } \\
\text { few millimeters to } 0.5 \mathrm{~cm} \text { maintaining contact with the cervix). The fingers should be facing } \\
\text { as in step } 9 .\end{array}$ \\
\hline 12 & $\begin{array}{l}\text { Now elevate the finger(s) by flexing the proximal or intermediate interphalangeal joint (by } \\
\text { approximately } 40 \text { degrees) and thus lifting the cervix to elicit cervical excitation (some } \\
\text { choose to press on the surface of the cervix and os as an alternative). }\end{array}$ \\
\hline 13 & Allow the finger(s) to relax to their original position and repeat step 12 twice or more. \\
\hline 14 & Return the finger(s) to the position in step 11. \\
\hline 15 & At the same time as performing steps $11-14$, place the nondominant hand on the \\
\hline
\end{tabular}

This article is protected by copyright. All rights reserved. 


\begin{tabular}{|c|c|}
\hline & $\begin{array}{l}\text { abdomen, usually starting at the umbilicus, and palpate toward the pubic synthesis until } \\
\text { the fundus of the uterus is felt. }\end{array}$ \\
\hline 16 & $\begin{array}{l}\text { Simultaneously to step } 15, \text { using the finger(s) in the vagina elevate the cervix by flexing } \\
\text { the proximal interphalangeal joint and by association the uterus to bring them into the } \\
\text { palpating abdominal hand, thus stabilizing the pelvic organs between both hands } \\
\text { (assessing size, shape, and mobility). }\end{array}$ \\
\hline 17 & $\begin{array}{l}\text { With the uterus stabilized move both hands anteriorly and posteriorly to assess the } \\
\text { mobility of the uterus (balloting). }\end{array}$ \\
\hline 18 & Repeat step 17 twice or more. \\
\hline 19 & Move the abdominal hand into the left iliac fossa. \\
\hline 20 & $\begin{array}{l}\text { Supinate the finger(s) aiming for the left lateral fornix and angle the finger(s) laterally up to } \\
45 \text { degrees. }\end{array}$ \\
\hline 21 & $\begin{array}{l}\text { Insert the finger(s) into the left lateral fornix aiming for the abdominal fingers in the left iliac } \\
\text { fossa (also assess for adnexal tenderness). }\end{array}$ \\
\hline 22 & Aim the finger(s) and hand toward each other as if they are to meet. \\
\hline 23 & In the process feel for the ovary (not always felt). \\
\hline 24 & $\begin{array}{l}\text { Once the ovary is stabilized between both finger(s) and hand, assess size, shape, and } \\
\text { density, then move it anterior and posterior to assess stability and motion. }\end{array}$ \\
\hline 25 & $\begin{array}{l}\text { Once complete, mirror the same assessment (steps 19-24) on the right iliac fossa and } \\
\text { right lateral fornix. }\end{array}$ \\
\hline 26 & $\begin{array}{l}\text { Once complete, return the fingers to position in step } 11 \text { while returning the abdominal } \\
\text { hand to cup the fundus of the uterus suprapubically. }\end{array}$ \\
\hline 27 & $\begin{array}{l}\text { If endometriosis is suspected, insert the finger(s) deeper until the posterior fornix is felt } \\
\text { and attempt to feel the uterosacral ligaments. }\end{array}$ \\
\hline 28 & $\begin{array}{l}\text { Move the finger(s) anterior and posterior along the ligaments (feel for thickening and/or } \\
\text { nodularity). }\end{array}$ \\
\hline 29 & $\begin{array}{l}\text { Once complete, retract and rotate the finger(s) into the sagittal plane (with the index finger } \\
\text { above the middle finger if used) and remove finger(s) in the anteroposterior diameter. }\end{array}$ \\
\hline 30 & $\begin{array}{l}\text { After removing finger(s) from the vagina, inspect for blood and/or discharge and be } \\
\text { attentive to any abnormal odors. }\end{array}$ \\
\hline
\end{tabular}

This article is protected by copyright. All rights reserved. 
Table 2. Confusion matrix: frequency of scores by both raters who observed 10 videos and judged whether each of the 30 steps were clearly seen/verbalized, unclear, or not seen/verbalized.

\begin{tabular}{|l|l|l|l|l|l|}
\hline \multirow{2}{*}{} & \multicolumn{2}{|l|}{ Rater 2 } & \multicolumn{2}{l|}{} \\
\cline { 3 - 6 } & No & Unclear & Yes & Total \\
\hline \multirow{3}{*}{ Rater 1 } & No & 18 & 33 & 21 & 72 \\
\cline { 2 - 7 } & Unclear & 1 & 3 & 6 & 10 \\
\cline { 2 - 6 } & Yes & 1 & 31 & 186 & 218 \\
\hline Total & & 20 & 67 & 213 & 300 \\
\hline
\end{tabular}

Figure legends

Figure 1. Prototype pelvic model used to perform bimanual vaginal examination.

Figure 2. Side view of the pelvic model showing anterior and lateral view integrated cameras.

Figure 3. Left image: Percentage of the 30 CTA steps performed by 10 experts, according to Rater 1 (OM, light green) and Rater 2 (dark green). Right image: Percentage of the experts that performed each step of the 30-step task analysis, according to Rater 1 (OM, light green) and Rater 2 (dark green).

\section{Supporting information}

\section{Eighty-eight step cognitive task analysis}

This article is protected by copyright. All rights reserved. 


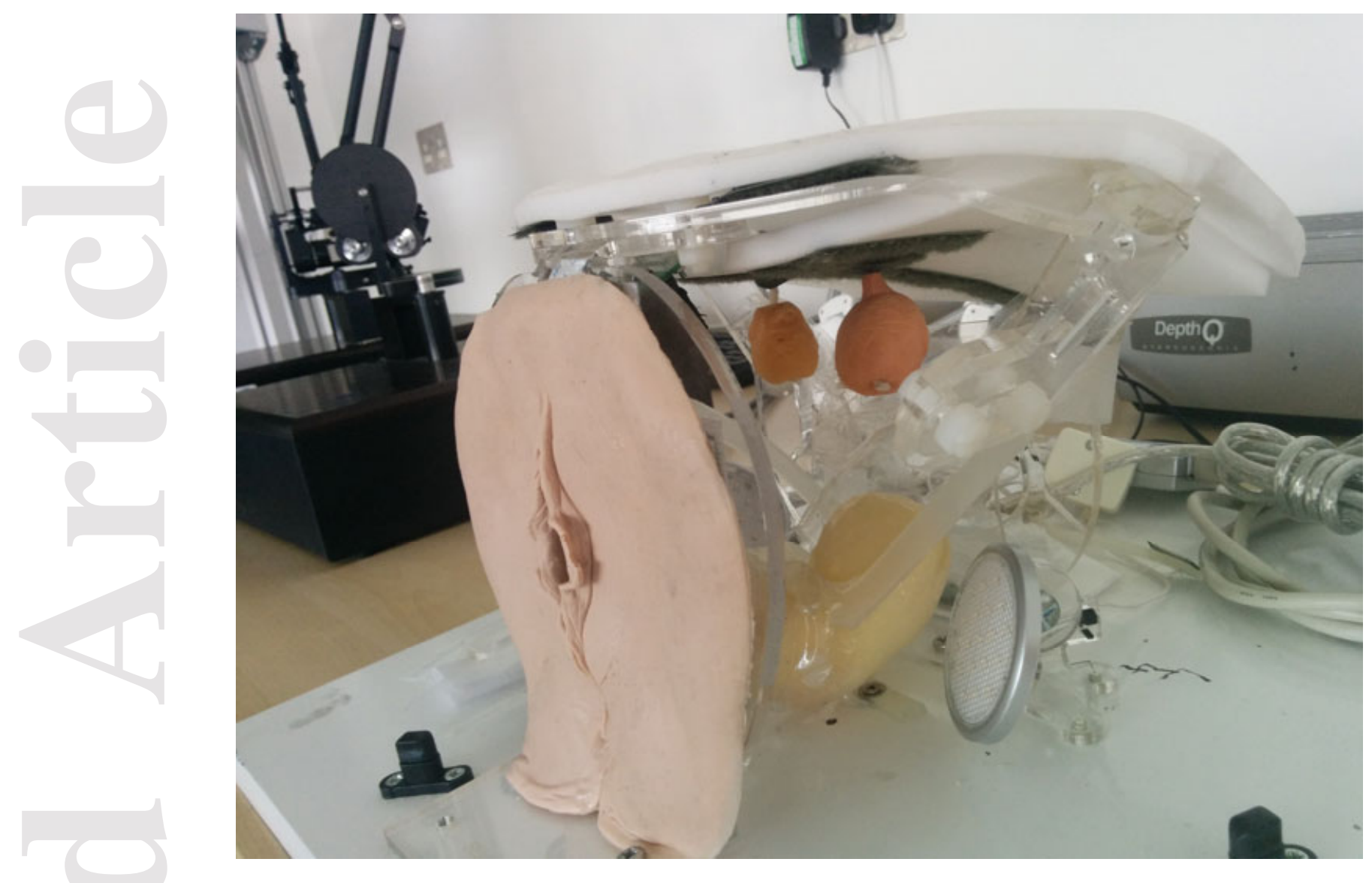

This article is protected by copyright. All rights reserved. 

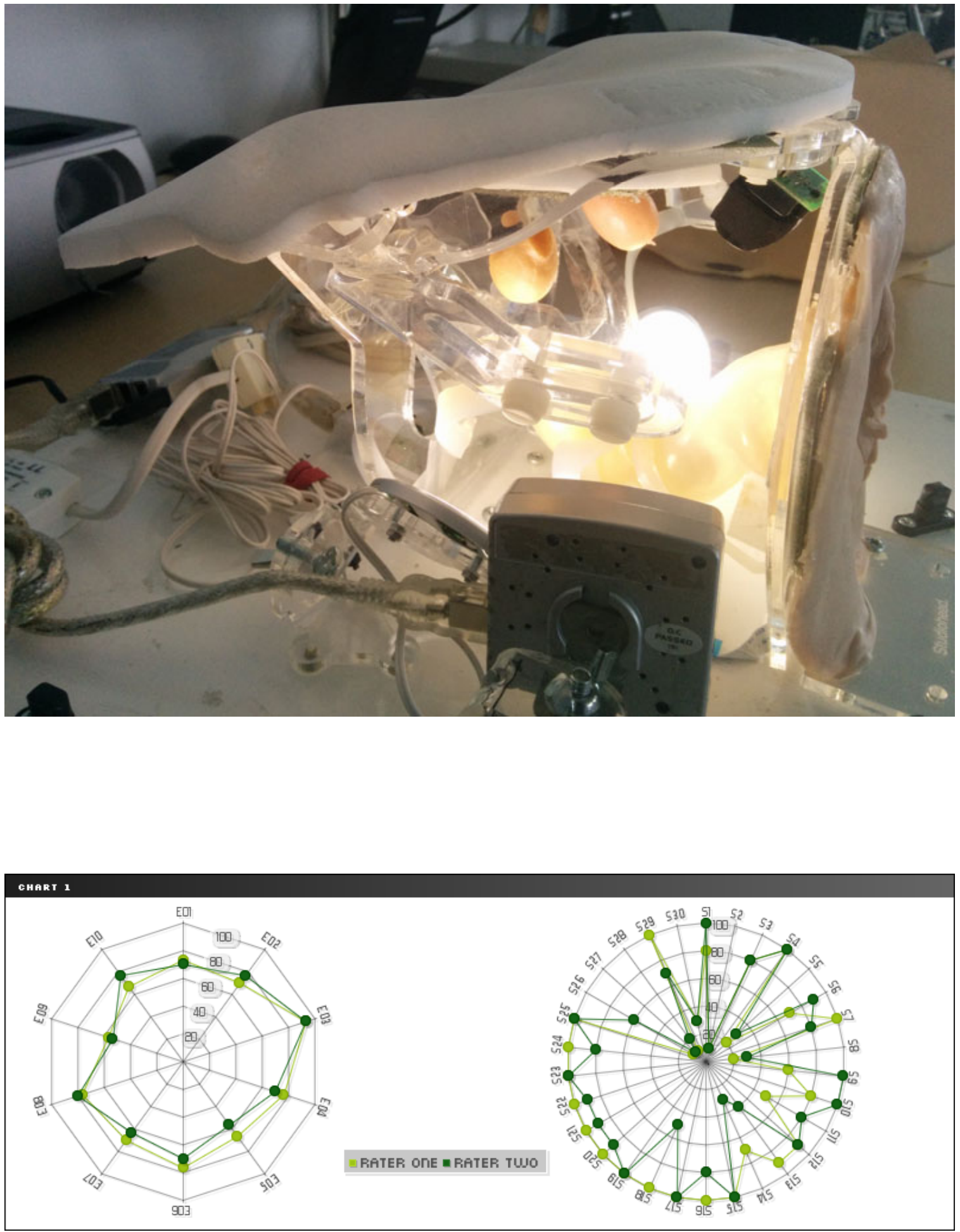

This article is protected by copyright. All rights reserved. 